\title{
Computing the Multiplicity Structure from Geometric Involutive Form
}

\author{
Xiaoli $\mathrm{Wu}^{\dagger}$ and Lihong Zhi ${ }^{\ddagger}$ \\ Key Laboratory of Mathematics Mechanization \\ AMSS,Academia Sinica, Beijing 100080, China \\ †xlwu@amss.ac.cn, ${ }^{\ddagger}$ lzhi@mmrc.iss.ac.cn
}

\begin{abstract}
We present a method based on symbolic-numeric reduction to geometric involutive form to compute the primary component and the differential operators for an isolated singular solution of a polynomial ideal. The singular solution can be exact or approximate. If the singular solution is known with limited accuracy, then we propose a new method to refine it to high accuracy.
\end{abstract}

\section{Introduction}

Consider a polynomial system $F=\left\{f_{1}, \ldots, f_{t}\right\}$, where $f_{i} \in \mathbb{C}\left[x_{1}, \ldots, x_{s}\right], i=1, \ldots, t$. For a given isolated singular solution $\hat{\mathbf{x}}=\left(\hat{x}_{1}, \ldots, \hat{x}_{s}\right)$ of $F$, suppose $Q$ is the isolated primary component whose associate prime is $P=\left(x_{1}-\hat{x}_{1}, \ldots, x_{s}-\hat{x}_{s}\right)$, we use a symbolic-numeric method based on the geometric jet theory of partial differential equations introduced in [35, $36,42]$ to compute the index $\rho$, such that $Q=\left(I, P^{\rho}\right)$. The multiplication structure of the quotient ring $\mathbb{C}[\mathbf{x}] / \mathbf{Q}$ is computed from the null space of the involutive form of $Q$. The differential operators are determined by computing the normal form of a polynomial with undetermined coefficients up to degree $\rho-1$. If the singular solution is only known with limited accuracy, then the primary ideal $Q$ has a cluster of solutions. A refined solution with higher accuracy can be obtained by averaging the eigenvalues of each multiplication matrix [5].

Inspired by recent works in $[9,8]$, we apply the involutive criterion to the matrices formulated from the coefficients of the truncated polynomials $T_{k}\left(f_{1}, \ldots, f_{t}\right)$ and their truncated prolongations. The number of columns of these coefficient matrices is fixed by $\left(\begin{array}{c}k+s-1 \\ s\end{array}\right)$. The differential operators can be obtained from the null space of the involutive system of $T_{\rho}\left(f_{1}, \ldots, f_{t}\right)$. Our algorithm for computing differential operators could be regarded as a primal version of the one given in [9].

Our method for computing index and multiplicity is also related to the one presented in [2]. The algorithm they presented for computing multiplicity is based on Bayer and Stillman's theory on regularity [3]. It was pointed out in [20] that the concept of involutivity of symbol is equivalent to its Mumford regularity. Our criterion for involutivity is similar to their stopping criterion for regularity. However we do not need homogenization procedure while the algorithm in [2] works for homogenous polynomials.

If a singular solution is only known with limited accuracy, by choosing a tolerance, we can compute the index, multiplicity and differential operators for this approximate singular 
solution. It is well known that numeric computations deeply depend on the choice of tolerance. In order to obtain accurate information about the multiplicity structure, we propose a method to improve the accuracy of the singular root first.

Suppose $\hat{\mathbf{x}}=\hat{\mathbf{x}}_{\text {exact }}+\hat{\mathbf{x}}_{\text {error }}$ where $\hat{\mathbf{x}}_{\text {exact }}$ denotes the exact singular solution of $F$. We observe that a good approximation $\hat{\mathbf{y}}$ of $-\hat{\mathbf{x}}_{\text {error }}$ can be computed from the involutive system of $T_{\rho+1}\left(f_{1}, \ldots, f_{t}\right)$. The singular solution $\hat{\mathbf{x}}+\hat{\mathbf{y}}$ has higher accuracy compared with $\hat{\mathbf{x}}$. We can apply our procedure iteratively to $\hat{\mathbf{x}}+\hat{\mathbf{y}}$ with a smaller tolerance. An accurate singular solution can usually be obtained in less than 3 iterations, as is shown in our experiments. It is still not clear how our refinement procedure related to the methods in [17, 31, 18, 32, 19]. The column dimension of the matrix we used for refining the approximate singular solution is $\left(\begin{array}{c}\rho+s \\ s\end{array}\right)$. Our algorithm for refining an approximate singular solution is not very efficient when index $\rho$ is very big. We notice that the number of deflations of the algorithms in $[18,19]$ is not closely related to the index, their algorithms can be very efficient for singular solutions with large index.

All algorithms we present in this paper have been implemented by the authors in Maple 11. We give two examples to illustrate our methods along the paper. We also show the test results for a set of benchmark problems. All computations are done in Maple 11 with Digits $:=14$.

\section{Isolated Primary Component}

\subsection{Preliminaries}

The following paragraphs give a brief outline of the notations and tools we use throughout this paper. We refer to $[6,40]$ for detailed introduction.

Definition 1 Let $I$ be an ideal in the ring of polynomials over complex field denoted by $\mathbb{C}[\mathbf{x}]=\mathbb{C}\left[\mathbf{x}_{1}, \ldots, \mathbf{x}_{\mathbf{s}}\right]$. Let $f, g$ be arbitrary elements in $\mathbb{C}[\mathbf{x}]$.

- $I$ is prime if $f g \in I \Longrightarrow f \in I$ or $g \in I$.

- I is primary if $f g \in I \Longrightarrow f \in I$ or $g^{m} \in I$ for some positive integer $m$.

- $I$ is radical if $f^{m} \in I \Longrightarrow f \in I$.

- The radical of $I$ is the set $\sqrt{I}=\left\{f \mid f^{m} \in I\right.$ for some integer $\left.m \geq 1\right\}$.

It should be noted that $\sqrt{I}$ is an ideal. Every prime ideal is a radical ideal and the radical of a primary ideal is a prime ideal. An ideal is finitely generated if there exists a finite list of elements $f_{1}, f_{2}, \ldots, f_{t} \in I$ such that every element in $I$ can be written as a $\mathbb{C}[\mathbf{x}]$-linear combination of $f_{1}, f_{2}, \ldots, f_{t}$, and denoted by $I=\left(f_{1}, f_{2}, \ldots, f_{t}\right)$.

Definition 2 If $P$ and $Q$ are ideals and have the property that

1. $f g \in Q$ and $f \notin Q$ implies $g \in P$,

2. $Q \subseteq P$,

3. $g \in P$ implies $g^{\rho} \in Q$ for some positive integer $\rho$,

\footnotetext{
${ }^{2)}$ In this paper, an accurate result is defined as having at least 14 correct digits
} 
then $Q$ is primary and $P$ the prime ideal belonging to $Q$.

If $Q$ is a primary ideal then $P=\sqrt{Q}$ is the prime ideal belonging to $Q$ and $Q$ is called $P$-primary.

Definition 3 Every polynomial ideal has an irredundant primary decomposition, i.e.

$$
I=\cap_{i=1}^{r} Q_{i}
$$

where $Q_{i}$ are primary, $Q_{i} \varsubsetneqq \cap_{j \neq i} Q_{j}$. We call $Q_{i}$ a primary component (ideal) of $I$. $Q_{i}$ is said to be isolated if no prime ideal belonging to $\cap_{j \neq i}^{r} Q_{j}$ is divisible by a prime ideal belonging to $Q_{i}$.

Definition $4 \rho$ is called the index of a primary ideal $Q$ if $\rho$ is the minimal nonnegative integer such that $\sqrt{Q}^{\rho} \subseteq Q$.

Theorem 1 [40] Suppose the polynomial ideal I has an isolated primary component $Q$ whose associated prime $P$ is maximal, and $\rho$ is the index of $Q$.

If $\sigma<\rho$, then

$$
\operatorname{dim}\left(\mathbb{C}[\mathbf{x}] /\left(\mathbf{I}, \mathbf{P}^{\sigma-\mathbf{1}}\right)\right)<\operatorname{dim}\left(\mathbb{C}[\mathbf{x}] /\left(\mathbf{I}, \mathbf{P}^{\sigma}\right)\right)
$$

If $\sigma \geq \rho$, then

$$
Q=\left(I, P^{\rho}\right)=\left(I, P^{\sigma}\right) .
$$

Corollary 1 If a polynomial ideal I has an isolated primary component $Q$ whose associated prime $P$ is maximal, then the index $\rho$ of $Q$ is less than or equal to the multiplicity of $Q$.

Proof: The multiplicity $\mu$ of the isolated primary component $Q$ is equal to the dimension of the quotient algebra $\mathbb{C}[\mathbf{x}] /\left(\mathbf{I}, \mathbf{P}^{\rho}\right)$. Since the dimension of $\mathbb{C}[\mathbf{x}] /\left(\mathbf{I}, \mathbf{P}^{\sigma}\right)$ increases strictly until $\sigma=\rho$, the multiplicity $\mu$ is bigger than or equal to the index $\rho$.

\subsection{SNEPSolver}

Consider a polynomial system $F=\left\{f_{1}, \ldots, f_{t}\right\}$, where $f_{i} \in \mathbb{C}[\mathbf{x}]$ is of degree $d, i=1, \ldots, t$ and $s \leq t$. The system can be written as

$$
M_{d}^{(0)} \cdot\left[x_{1}^{d}, x_{1}^{d-1} x_{2}, \ldots, x_{s}^{2}, x_{1}, \ldots, x_{s}, 1\right]^{T}=[0,0, \ldots, 0,0, \ldots, 0,0]^{T}
$$

in terms of its coefficient matrix $M_{d}^{(0)}$. Here and hereafter, $[\ldots]^{T}$ means the transposition. Further, $\left[\xi_{1}, \xi_{2}, \ldots, \xi_{s}\right]$ is one of the solutions of the polynomial system, if and only if

$$
\left[\xi_{1}^{d}, \xi_{1}^{d-1} \xi_{2}, \ldots, \xi_{s}^{2}, \xi_{1}, \ldots, \xi_{s}, 1\right]^{T}
$$

is a null vector of the coefficient matrix $M_{d}^{(0)}$.

Since the number of monomial terms is usually much greater than the number of polynomials, the dimension of the null space can be large. Completion methods for polynomial ideals based on critical pairs $[16,10,11,23,1,24,38,27,28,29,39,30]$ aim to include additional polynomials belonging to the ideal generated by $F$, until the normal form is determined 
capable of deciding membership of the ideal. The method in [34, 35, 36, 42] focuses on direct methods to calculate and minimize these dimensions without using critical pair techniques but using the criterion of involution for PDE $R[14,33]$. Here $R$ is equivalent to polynomial system $F$ by the bijection

$$
\phi: x_{i} \leftrightarrow \frac{\partial}{\partial x_{i}}, \quad 1 \leq i \leq s
$$

In the following, we briefly explain the symbolic-numeric elimination method in the language of polynomial algebra. We study the variety

$$
V(F)=\left\{\left[\mathbf{x}^{\mathbf{d}}, \ldots, \mathbf{x}^{\mathbf{1}}, \mathbf{1}\right] \in \mathbb{C}^{\mathbf{N}_{\mathbf{d}}} \mid \mathbf{M}_{\mathbf{d}}^{(\mathbf{0})} \cdot\left[\mathbf{x}^{\mathbf{d}}, \mathbf{x}^{\mathbf{d}-\mathbf{1}}, \ldots, \mathbf{x}^{\mathbf{1}}, \mathbf{1}\right]^{\mathbf{T}}=\mathbf{0}\right\} .
$$

A single prolongation of the system $F$ is to multiply the polynomials in $F$ by variables, so that the resulting augmented system has degree $d+1$. Successive prolongations of the system yield $F, F^{(1)}, F^{(2)}, \ldots$, and a sequence of corresponding linear constant matrix systems:

$$
M_{d}^{(0)} \cdot \mathbf{v}_{\mathbf{d}}=\mathbf{0}, \mathbf{M}_{\mathbf{d}}^{(\mathbf{1})} \cdot \mathbf{v}_{\mathbf{d}+\mathbf{1}}=\mathbf{0}, \mathbf{M}_{\mathbf{d}}^{(2)} \cdot \mathbf{v}_{\mathbf{d}+\mathbf{2}}=\mathbf{0}, \cdots
$$

where $\mathbf{v}_{\mathbf{i}}=\left[\mathbf{x}^{\mathbf{i}}, \mathbf{x}^{\mathbf{i}-\mathbf{1}}, \ldots, \mathbf{x}, \mathbf{1}\right]^{\mathbf{T}}, \mathbf{x}^{\mathbf{j}}$ denotes all monomials of total degree equal to $j$.

A single geometric projection is defined as

$$
\boldsymbol{\pi}(F):=\left\{\left[\mathbf{x}^{\mathbf{d}-1}, \ldots, \mathbf{x}^{\mathbf{1}}, \mathbf{1}\right] \in \mathbb{C}^{\mathbf{N}_{\mathbf{d}-1}} \mid \exists \mathbf{x}^{\mathrm{d}}, \mathbf{M}_{\mathbf{d}}^{(\mathbf{0})} \cdot\left[\mathbf{x}^{\mathbf{d}}, \mathbf{x}^{\mathbf{d}-1}, \ldots, \mathbf{x}^{\mathbf{1}}, \mathbf{1}\right]^{\mathbf{T}}=\mathbf{0}\right\} .
$$

The projection operator $\boldsymbol{\pi}$ maps a point in $\mathbb{C}^{N_{d}}$ where $N_{d}=\left(\begin{array}{c}d+s \\ s\end{array}\right)$ to one in $\mathbb{C}^{N_{d-1}}$ by eliminating the monomials of the highest degree $d$. A numeric projection operator $\hat{\boldsymbol{\pi}}$ based on singular value decomposition (SVD) was purposed in $[4,41,35]$. We first find the SVD of $M_{d}^{(0)}$ :

$$
M_{d}^{(0)}=U \cdot \Sigma \cdot V
$$

The approximate rank $r$ of $M_{d}^{(0)}$ is the number of singular values bigger than a fixed tolerance. The tolerance is chosen according to the number of correct digits for the coefficients of the input polynomials. The dimension of the null space of $M_{d}^{(0)}$ is denoted by $\operatorname{dim}(F)=$ $N_{d}-r$. Deleting the first $r$ rows of $V$ yields an approximate basis for the null space of $M_{d}^{(0)}$. To estimate $\operatorname{dim}(\hat{\boldsymbol{\pi}}(F))$, the components of the approximate basis for the null space of $M_{d}^{(0)}$ corresponding to highest degree $d$ th monomials are deleted. This projected basis yields an approximate spanning set for $\hat{\boldsymbol{\pi}}(F)$. Application of the SVD to each of these approximate spanning sets yields the approximate dimensions of $\hat{\boldsymbol{\pi}}(F), \hat{\boldsymbol{\pi}}^{2}(F), \hat{\boldsymbol{\pi}}^{3}(F), \ldots$, which are required for the approximate involutive form test.

The symbol matrix of polynomials of degree $d$ is simply the submatrix of the coefficient matrix $M_{d}^{(0)}$ corresponding to highest degree $d$ th monomials. One of the most important requirements of involutive systems is that their symbols are involutive.

Theorem 2 (Criterion of involution for zero dimensional polynomial systems) [42] $A$ zero dimensional polynomial system $F$ is involutive at order $m$ and projected order $\ell$ if and only if $\boldsymbol{\pi}^{\ell}\left(F^{(m)}\right)$ satisfies the projected elimination test

$$
\operatorname{dim} \boldsymbol{\pi}^{\ell}\left(F^{(m)}\right)=\operatorname{dim} \boldsymbol{\pi}^{\ell+1}\left(F^{(m+1)}\right)
$$


and the symbol involutive test:

$$
\operatorname{dim} \boldsymbol{\pi}^{\ell}\left(F^{(m)}\right)=\operatorname{dim} \boldsymbol{\pi}^{\ell+1}\left(F^{(m)}\right) .
$$

If the case (10) holds then $\operatorname{dim}\left(\operatorname{Symbol} \pi^{\ell}\left(F^{(m)}\right)\right)=0$. It is easily shown that the symbol of $\boldsymbol{\pi}^{\ell}\left(F^{(m)}\right)$ is involutive [3r].

The following algorithm given in $[42,36]$ solves zero dimensional polynomial systems based on the symbolic-numeric completion method.

\section{Algorithm 1 SNEPSolver}

Input: $A$ zero dimensional ideal $I=\left(f_{1}, \ldots, f_{t}\right)$ where the polynomials are in $\mathbb{C}[\mathbf{x}]$ of degree $d$ and a tolerance $\tau$.

Output: $\operatorname{dim}(\mathbb{C}[\mathbf{x}] / \mathbf{I})$ and multiplication matrices $M_{x_{1}}, \ldots, M_{x_{s}}$ of the quotient ring $\mathbb{C}[\mathbf{x}] / \mathbf{I}$.

- Apply the symbolic-numeric completion method to $F=\left\{f_{1}, \ldots, f_{t}\right\}$ with tolerance $\tau$, we obtain the table of dimensions of $\operatorname{dim} \hat{\pi}^{\ell}\left(F^{(m)}\right)$.

- We seek the smallest $m$ such that there exists an $\ell$ with $\hat{\pi}^{\ell}\left(F^{(m)}\right)$ approximately involutive, i.e., satisfying the conditions (9, 10). If there are several such values for the given $m$, then choose the largest such $\ell$.

- The number of solutions of polynomial system $F$ is

$$
\operatorname{dim}(\mathbb{C}[\mathbf{x}] / \mathbf{I})=\operatorname{dim} \hat{\boldsymbol{\pi}}^{\ell}\left(\mathbf{F}^{(\mathbf{m})}\right) .
$$

- The multiplication matrices $M_{x_{1}}, \ldots, M_{x_{s}}$ are formed from the null vectors of $\hat{\boldsymbol{\pi}}^{\ell}\left(F^{(m)}\right)$ and $\hat{\boldsymbol{\pi}}^{\ell+1}\left(F^{(m)}\right)$.

The solutions of $F$ can be obtained by computing eigenvalues and eigenvectors of the multiplication matrices $[1,5,24]$.

\subsection{Algorithm for Computing Isolated Primary Component}

For a given isolated solution of the ideal $I=\left(f_{1}, \ldots, f_{t}\right)$, suppose $Q$ is the isolated primary component whose associate prime $P=\left(x_{1}-\hat{x}_{1}, \ldots, x_{s}-\hat{x}_{s}\right)$, we apply the Algorithm SNEPSolver to compute the index $\rho$, such that $Q=\left(I, P^{\rho}\right)$ and the multiplication structure of the quotient ring $\mathbb{C}[\mathbf{x}] / \mathbf{Q}$.

Algorithm 2 IsolatedPrimaryComponent

Input: An isolated multiple solution $\hat{\mathbf{x}}$ of an ideal $I=\left(f_{1}, \ldots, f_{t}\right)$, a tolerance $\tau$.

Output: The multiplicity $\mu$, the index $\rho$, and multiplication matrices $M_{x_{1}}, \ldots, M_{x_{s}}$ of the quotient ring $\mathbb{C}[\mathbf{x}] / \mathbf{Q}$ where $Q=\left(I, P^{\rho}\right)$.

- Form the associate prime ideal $P=\left(x_{1}-\hat{x}_{1}, \ldots, x_{s}-\hat{x}_{s}\right)$.

- Compute $d_{k}=\operatorname{dim}\left(\mathbb{C}[\mathbf{x}] /\left(\mathbf{I}, \mathbf{P}^{\mathbf{k}}\right)\right)$ as described above by SNEPSolver until $d_{k}=d_{k-1}$, then set $\rho=k-1, \mu=d_{\rho}$ and $Q=\left(I, P^{\rho}\right)$. 
- Compute the multiplication matrices $M_{x_{1}}, \ldots, M_{x_{s}}$ of $\mathbb{C}[\mathbf{x}] / \mathbf{Q}$ by SNEPSolver.

Symbolic methods based on the uniqueness of the reduced Gröbner basis are given in $[12,15]$ to determine the index of $Q$. However, when the multiple zero is only known with finite precision, their methods are subject to numerical stability problem.

Remark 1 The set made up of these computed multiplication matrices

$$
\left\{M_{x_{1}}, \ldots, M_{x_{s}}\right\}
$$

is called numerical local ring in [8] for a given root $\hat{\mathbf{x}}$.

Since the ideal $\left(I, P^{k}\right)$ is generated by polynomials

$$
F_{k}=\left\{f_{1}, \ldots, f_{t},\left(x_{1}-\hat{x}_{1}\right)^{\alpha_{1}} \cdots\left(x_{s}-\hat{x}_{s}\right)^{\alpha_{s}}, \alpha_{1}+\cdots+\alpha_{s}=k\right\} .
$$

Without loss of generality, suppose $d \leq k$, we prolong all polynomials $f_{i}$ to have degree $k$. Since all monomials of degree $k+j$ appear in the prolonged system $F_{k}^{(j)}$, the symbol matrices of $F_{k}^{(j)}$ always have full rank, i.e., the symbols of $F_{k}^{(j)}$ are involutive. The dimension of the null space of the prolonged system decreases strictly until it is stabilized. So we have a simple criterion for involution of $F_{k}$.

Theorem 3 (Criterion of involution for polynomial system $F_{k}$ ) The zero dimensional polynomial system $F_{k}$ is involutive at order $m$ if and only if

$$
\operatorname{dim} F_{k}^{(m)}=\operatorname{dim} F_{k}^{(m+1)}=d_{k}=\operatorname{dim}\left(\mathbb{C}[\mathbf{x}] /\left(\mathbf{I}, \mathbf{P}^{\mathbf{k}}\right)\right) .
$$

Example 1 [31] Consider an ideal I generated by the polynomials

$$
\left\{f_{1}=x_{1}^{2}+x_{2}-3, f_{2}=x_{1}+0.125 x_{2}^{2}-1.5\right\} .
$$

The system has $(1,2)$ as a 3 -fold solution.

Form the maximal ideal $P=\left(x_{1}-1, x_{2}-2\right)$.

- $k=2$, we consider the system

$$
F_{2}=\left\{f_{1}, f_{2},\left(x_{1}-1\right)^{2},\left(x_{1}-1\right)\left(x_{2}-2\right),\left(x_{2}-2\right)^{2}\right\} .
$$

Since $\operatorname{dim}\left(F_{2}\right)=1, \operatorname{dim}\left(F_{2}^{(1)}\right)=\operatorname{dim}\left(F_{2}^{(2)}\right)=2$, we have $\operatorname{dim}\left(\mathbb{C}[\mathbf{x}] /\left(\mathbf{I}, \mathbf{P}^{\mathbf{2}}\right)\right)=\mathbf{2}$.

Similarly, we have

- $k=3, \operatorname{dim}\left(F_{3}\right)=1, \operatorname{dim}\left(F_{3}^{(1)}\right)=\operatorname{dim}\left(F_{3}^{(2)}\right)=3, \operatorname{dim}\left(\mathbb{C}[\mathbf{x}] /\left(\mathbf{I}, \mathbf{P}^{\mathbf{3}}\right)\right)=\mathbf{3}$.

- $k=4, \operatorname{dim}\left(F_{4}\right)=1, \operatorname{dim}\left(F_{4}^{(1)}\right)=\operatorname{dim}\left(F_{4}^{(2)}\right)=3, \operatorname{dim}\left(\mathbb{C}[\mathbf{x}] /\left(\mathbf{I}, \mathbf{P}^{4}\right)\right)=\mathbf{3}$. 
Therefore, index and multiplicity of the root $(1,2)$ are: $\rho=3, \mu=3$. The multiplication matrices are:

$$
M_{x_{1}}=\left[\begin{array}{ccc}
0 & -1 & 3 \\
6 & 3 & -10 \\
1 & 0 & 0
\end{array}\right], M_{x_{2}}=\left[\begin{array}{ccc}
6 & 3 & -10 \\
-8 & 0 & 12 \\
0 & 1 & 0
\end{array}\right]
$$

The triple eigenvalues of $M_{x_{1}}$ and $M_{x_{2}}$ are 1 and 2 respectively.

If the singular solution $\hat{\mathbf{x}}$ is only known approximately, then the polynomial system $F_{k}$ has a cluster of solutions. The Schur factorization of multiplication matrices $M_{x_{i}}$ consists of only one block. As shown in [5], the average of the cluster eigenvalues of $M_{x_{i}}$ computed by $\operatorname{Trace}\left(M_{x_{i}}\right) / \mu$ gives a refined value for $\hat{x}_{i}$. We can apply the procedure again for the refined singular solution and obtain singular solution with higher accuracy.

Example 1 (continued) Suppose we are given an approximate singular solution:

$$
\hat{\mathbf{x}}=\left(1+2.5428 \times 10^{-4}+2.4352 \times 10^{-4} i, 2+8.4071 \times 10^{-4}+3.6129 \times 10^{-4} i\right) .
$$

We choose the tolerance $\tau=10^{-4}$ and apply the Algorithm 2 to $\hat{\mathbf{x}}$ and the polynomial system (14). The dimensions computed for the given tolerance are the same as shown above. Therefore, we get the same index and multiplicity for the approximate singular solution with respect to the given tolerance. The refined root computed from the multiplication matrices is:

$$
\left(1+9.5829 \times 10^{-8}-1.2762 \times 10^{-7} i, 2-2.6679 \times 10^{-6}+3.5569 \times 10^{-7} i\right) .
$$

Then use this refined solution as an initial solution and set $\tau=10^{-6}$, run the Algorithm 2 again, we obtain:

$$
\left(1-1.0000 \times 10^{-15}+2.5854 \times 10^{-14} i, 2+8.4457 \times 10^{-14}\right) .
$$

\section{Modified SNEPSolver for Computing Differential Operators}

Let $D(\alpha)=D\left(\alpha_{1}, \ldots, \alpha_{s}\right): \mathbb{C}[\mathbf{x}] \rightarrow \mathbb{C}[\mathbf{x}]$ denote the differential operator defined by:

$$
D\left(\alpha_{1}, \ldots, \alpha_{s}\right)=\frac{1}{\alpha_{1} ! \cdots \alpha_{s} !} \partial x_{1}^{\alpha_{1}} \cdots \partial x_{s}^{\alpha_{s}}, \quad \alpha_{j} \in \mathbb{N} .
$$

We write $\mathfrak{D}=\{\mathfrak{D}(\alpha),|\alpha| \geq \mathbf{0}\}$ and denote by $\operatorname{Span}_{\mathbb{C}}(\mathfrak{D})$ the $\mathbb{C}$-vector space generated by $\mathfrak{D}$ and introduce a morphism on $\operatorname{Span}_{\mathbb{C}}(\mathfrak{D})$ that acts as "integral":

$$
\sigma_{x_{j}}\left(D\left(\alpha_{1}, \ldots, \alpha_{s}\right)\right)= \begin{cases}D\left(\alpha_{1}, \ldots, \alpha_{j}-1, \ldots, \alpha_{s}\right), & \text { if } \alpha_{j}>0 \\ 0, & \text { otherwise. }\end{cases}
$$

Definition 5 A subspace $L$ of $\operatorname{Span}_{\mathbb{C}}(\mathfrak{D})$ is said to be closed if

$$
\sigma_{x_{j}}(L) \subseteq L, \text { for all } j=1, \ldots, s .
$$

Definition 6 Given a zero $\hat{\mathbf{x}}=\left(\hat{x}_{1}, \ldots, \hat{x}_{s}\right)$ of an ideal I, we define the subspace of differential operators associated to $I$ as

$$
\triangle_{\hat{\mathbf{x}}}:=\left\{L \in \operatorname{Span}_{\mathbb{C}}(\mathfrak{D}) \mid L(f)(\hat{\mathbf{x}})=0 \text { for all } f \in I\right\} .
$$


Theorem 4 Let $m$ be the maximal ideal $\left(x_{1}, \ldots, x_{s}\right)$ of $\mathbb{C}[\mathbf{x}]$. There is a bijective correspondence between $m$-primary ideals of $\mathbb{C}[\mathbf{x}]$ and closed subspaces of $\operatorname{Span}_{\mathbb{C}}(\mathfrak{D})$

$$
\{m \text {-primary ideals in } \mathbb{C}[\mathbf{x}]\} \rightleftharpoons\left\{\text { closed subspaces of } \operatorname{Span}_{\mathbb{C}}(\mathfrak{D})\right\} \text {. }
$$

Moreover, for a zero dimensional $m$-primary ideal of $\mathbb{C}[\mathbf{x}]$ whose multiplicity is $\mu$, we have that $\operatorname{dim}_{\mathbb{C}}\left(\triangle_{\hat{\mathbf{x}}}\right)=\mu$.

\subsection{Algorithm for Computing Differential Operators}

The following algorithm computes the differential operators from the output of the Algorithm 2 .

\section{Algorithm 3 DifferentialOperatorsI}

Input: Multiplication matrices $M_{x_{1}}, \ldots, M_{x_{s}}$, multiple zero $\hat{\mathbf{x}}$ and index $\rho$, a tolerance $\tau$.

Output: $L=\left\{L_{0}, \ldots, L_{\mu-1}\right\}$.

- Write the Taylor expansion at $\hat{\mathbf{x}}$ of a polynomial $h \in \mathbb{C}[\mathbf{x}]$ up to the degree $\rho-1$ with coefficients $c_{\alpha} \in \mathbb{C}$ :

$$
T_{\rho-1} h\left(x_{1}, \ldots, x_{s}\right)=\sum_{|\alpha|<\rho} c_{\alpha}\left(x_{1}-\hat{x}_{1}\right)^{\alpha_{1}} \cdots\left(x_{s}-\hat{x}_{s}\right)^{\alpha_{s}} .
$$

- Compute the normal form of $h$

$$
\mathrm{NF}(h(\mathbf{x}))=\mathbf{r} \cdot \mathbf{h}\left(\mathbf{M}_{\mathbf{x}_{1}}, \ldots, \mathbf{M}_{\mathbf{x}_{\mathbf{s}}}\right) \cdot \mathbf{q}=\sum_{\beta} \mathbf{d}_{\beta} \mathbf{x}^{\beta},
$$

where $\mathbf{q}$ is an basis of $\mathbb{C}[\mathbf{x}] / \mathbf{Q}$, and $\mathbf{r}$ is a vector satisfying $\mathbf{r} \cdot \mathbf{q}=\mathbf{1}$.

- Find scalars $a_{\alpha \beta} \in \mathbb{C}$ such that $d_{\beta}=\sum_{\alpha} a_{\alpha \beta} c_{\alpha}$. For each $\beta$ such that $d_{\beta} \neq 0$, return the operator

$$
L_{\beta}=\sum_{\alpha} a_{\alpha \beta} \frac{1}{\alpha_{1} ! \cdots \alpha_{s} !} \partial x_{1}^{\alpha_{1}} \cdots \partial x_{s}^{\alpha_{s}}=\sum_{\alpha} a_{\alpha \beta} D(\alpha) .
$$

An alternative procedure for computing these differential operators based on Gröbner basis computation is given in [7]. Our algorithm can be applied to polynomial system with floating point coefficients since it computes the normal form stably by using the Algorithm 1 SNEPSolver. Furthermore, we use the index as a bound which is tighter than the multiplicity used in [7].

Remark 2 For polynomial system with floating point coefficients, we can compute an orthogonal basis $\mathbf{q}=\mathbf{U} \cdot \mathcal{N}^{\mathcal{T}}$ of $\mathbb{C}[\mathbf{x}] / \mathbf{Q}$, and $\mathbf{r}$ is the Hermitian transpose of the last column of $U$ satisfying $\mathbf{r} \cdot \mathbf{q}=\mathbf{1}$. It should be noticed that the normal form of $h$ is computed by multiplying vector $\mathbf{r}$ to the multiplication matrices $M_{x_{1}}, \ldots, M_{x_{s}}$ first, so that we only perform vector and matrix multiplications.

Example 1 (continued) We compute the differential operators at the exact solution $(1,2)$ : 
- Write the Taylor expansion of a polynomial at $(1,2)$ up to degree $\rho-1=2$,

$$
h(\mathrm{x})=\mathrm{c}_{0,0}+\mathrm{c}_{1,0}\left(\mathrm{x}_{1}-1\right)+\mathrm{c}_{0,1}\left(\mathrm{x}_{2}-2\right)+\mathrm{c}_{2,0}\left(\mathrm{x}_{1}-1\right)^{2}+\mathrm{c}_{1,1}\left(\mathrm{x}_{1}-1\right)\left(\mathrm{x}_{2}-2\right)+\mathrm{c}_{0,2}\left(\mathrm{x}_{2}-2\right)^{2} .
$$

- Compute the normal form of $h$, and translate the three coefficients to differential operators

$$
\left\{\begin{array}{l}
L_{1}=D(0,0)-D(1,0)-2 D(0,1)+4 D(2,0)-8 D(1,1)+16 D(0,2) \\
L_{2}=D(0,1)-D(0,2)+2 D(1,1)-4 D(2,0) \\
L_{3}=D(1,0)-2 D(2,0)+4 D(1,1)-8 D(0,2)
\end{array}\right.
$$

Theorem 5 Let $\tilde{F}=\left\{L_{j}\left(f_{i}\right) \mid L_{j} \in L, f_{i} \in F\right\}$, then $\hat{\mathbf{x}}$ is an isolated simple solution of $\tilde{F}$.

Proof: If $\hat{\mathbf{x}}$ is a singular solution of $\tilde{F}$, then there exists a non-trivial differential operator $L_{\tau}$, its differential order $|\tau| \geq 1$. Take $L_{\beta} \in L$ that has the highest differential order in $L$. Then $L_{\tau} \circ L_{\beta}$ is a differential operator for $F$ at $\hat{\mathbf{x}}$ which is not included in $L$. It is a contradiction.

\subsection{Specialized SNEPSolver}

We have applied the Algorithms 2 and 3 to a set of examples shown in the Table 1. For some examples, the systems become too large after we add all monomials of large degrees. In this subsection, we propose a modified SNEPSolver. The matrices we used to verify the involutivity are much smaller than the ones used by SNEPSolver.

Suppose $\hat{\mathbf{x}}=\left(\hat{x}_{1}, \ldots, \hat{x}_{s}\right)$ is an isolated zero of a set of multivariate polynomials $F=$ $\left\{f_{1}, \ldots, f_{t}\right\}$. The zero can be moved to the origin by changing of variables. For simplicity, suppose $\hat{\mathbf{x}}$ is the origin. Let $P=\left(x_{1}, \ldots, x_{s}\right)$ and $I$ be an ideal having $P$-primary isolated component. Let

$$
\mathrm{T}_{k}(F)=\left\{\mathrm{T}_{k}\left(f_{1}\right), \ldots, \mathrm{T}_{k}\left(f_{t}\right)\right\},
$$

where $\mathrm{T}_{k}\left(f_{i}\right)=\sum_{|\alpha|<k} f_{i, \alpha} x^{\alpha}$ denotes the truncated polynomial of $f_{i}=\sum f_{i, \alpha} x^{\alpha}$.

The ideal $\left(I, P^{k}\right)$ is generated by the polynomials $F_{k}=\mathrm{T}_{k}(F) \cup P^{k}$. The symbol matrices of $F_{k}$ and prolonged system $F_{k}^{(j)}$ consist of an identity matrix generated by the coefficients of monomials in $P^{k}$. Therefore the symbol matrices are of full rank, and the dimensions and null spaces of coefficient matrices of the systems $F_{k}$ and $F_{k}^{(j)}$ can be computed from the coefficient matrices generated by the truncated systems $F_{k}$ and $\mathrm{T}_{k}\left(F^{(j)}\right)$. So we can work with the coefficient matrices of truncated system $\mathrm{T}_{k}(F)$ and truncated prolonged systems $\mathrm{T}_{k}\left(F^{(j)}\right)$ instead of the coefficient matrices of $F \cup P^{k}$ and their prolongations. For simplicity, we still use $M_{k}^{(j)}$ to denote the coefficient matrices of the system $\mathrm{T}_{k}\left(F^{(j)}\right)$.

Since all polynomials are truncated by degree $k$, the coefficient matrix $M_{k}^{(j)}$ has only $\left(\begin{array}{c}k+s-1 \\ s\end{array}\right)$ columns. Furthermore, the number of prolongations $m$ has an upper bound:

$$
m \leq \max \left(1, k-\min \left(\operatorname{ldeg}\left(f_{1}\right), \ldots, \operatorname{ldeg}\left(f_{t}\right)\right)\right) .
$$

$\operatorname{ldeg}(f)$ denotes the lowest degree of $f$.

The following example is used to illustrate the simplified SNEPSolver. 
Example 2 [19] We consider an ideal I generated by the polynomials

$$
\left\{f_{1}=x_{1}^{3}+x_{1} x_{2}^{2}, f_{2}=x_{1} x_{2}^{2}+x_{2}^{3}, f_{3}=x_{1}^{2} x_{2}+x_{1} x_{2}^{2}\right\} .
$$

The system has $(0,0)$ as a 7 -fold solution.

We form the maximal ideal $P=\left(x_{1}, x_{2}\right)$.

- $k=2$. The coefficient matrix $M_{2}^{(0)}$ is

$$
\begin{aligned}
& \mathrm{T}_{2}\left(f_{1}\right) \\
& \mathrm{T}_{2}\left(f_{2}\right) \\
& \mathrm{T}_{2}\left(f_{3}\right)
\end{aligned}\left[\begin{array}{lll}
0 & 0 & 0 \\
0 & 0 & 0 \\
0 & 0 & 0
\end{array}\right]\left[\begin{array}{c}
x_{1} \\
x_{2} \\
1
\end{array}\right]
$$

and $d_{2}^{(0)}=3$. The prolonged matrix $M_{2}^{(1)}$ is also a zero matrix. Hence $d_{2}^{(1)}=3$ and

$$
d_{2}=\operatorname{dim}\left(\mathbb{C}[\mathbf{x}] /\left(\mathbf{I}, \mathbf{P}^{\mathbf{2}}\right)\right)=\mathbf{3} .
$$

- $k=3$. The coefficient matrices $M_{3}^{(0)}$ and $M_{3}^{(1)}$ are zero matrices. We have

$$
d_{3}=\operatorname{dim}\left(\mathbb{C}[\mathbf{x}] /\left(\mathbf{I}, \mathbf{P}^{\mathbf{3}}\right)\right)=\mathbf{6} .
$$

- $k=4$. The coefficient matrix $M_{4}^{(0)}$ is

$$
\begin{aligned}
& \mathrm{T}_{4}\left(f_{1}\right) \\
& \mathrm{T}_{4}\left(f_{2}\right) \\
& \mathrm{T}_{4}\left(f_{3}\right)
\end{aligned}\left[\begin{array}{llllllllll}
1 & 0 & 1 & 0 & 0 & 0 & 0 & 0 & 0 & 0 \\
0 & 0 & 1 & 1 & 0 & 0 & 0 & 0 & 0 & 0 \\
0 & 1 & 1 & 0 & 0 & 0 & 0 & 0 & 0 & 0
\end{array}\right]\left[\begin{array}{c}
x_{1}^{3} \\
\vdots \\
x_{2} \\
1
\end{array}\right]
$$

and $d_{4}^{(0)}=7$. After the first prolongation, we get $M_{4}^{(1)}$

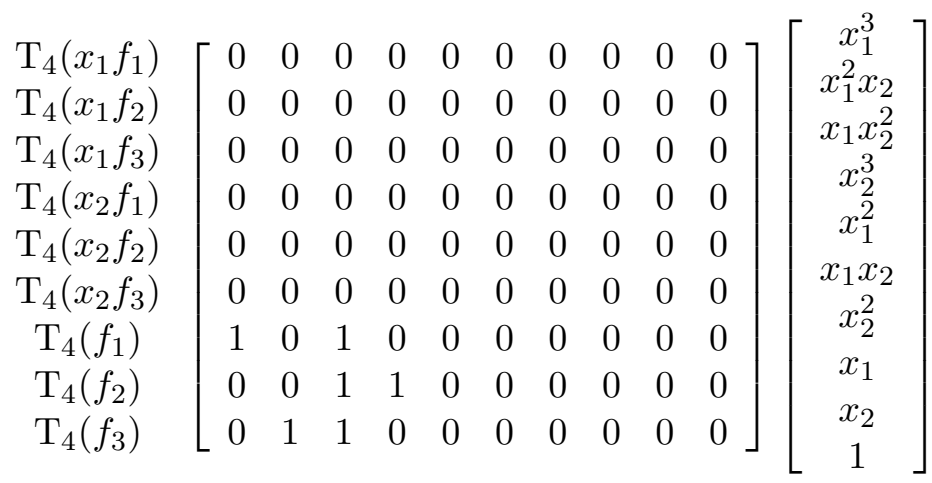

Since $d_{4}^{(1)}=d_{4}^{(0)}=7$, we have

$$
d_{4}=\operatorname{dim}\left(\mathbb{C}[\mathbf{x}] /\left(\mathbf{I}, \mathbf{P}^{\mathbf{4}}\right)\right)=\mathbf{7} .
$$


- $k=5$. We compute $d_{5}^{(0)}=12, d_{5}^{(1)}=d_{5}^{(2)}=7$. Hence,

$$
d_{5}=\operatorname{dim}\left(\mathbb{C}[\mathbf{x}] /\left(\mathbf{I}, \mathbf{P}^{\mathbf{5}}\right)\right)=\mathbf{7} .
$$

Since $d_{4}=d_{5}=7$, we obtain that the multiplicity of the solution $(0,0)$ is $\mu=7$ and the index of the primary ideal is $\rho=4$, i.e. $Q=\left(I, P^{4}\right)$.

The null space of the matrix $M_{4}^{(0)}$ has dimension 7 and can be written as

$$
N_{4}^{(0)}=\left[e_{1}, e_{1},-e_{1}, e_{1}, e_{2}, e_{3}, e_{4}, e_{5}, e_{6}, e_{7}\right]^{T},
$$

where $e_{i}$ is the $i$ th column of $7 \times 7$ identity matrix.

It is interesting to notice that the differential operators can be obtained by multiplying the differential operators of order less than 4 with the 7 null vectors in $N_{4}^{(0)}$ :

$$
\left\{\begin{array}{l}
D(3,0)+D(2,1)-D(1,2)+D(0,3), \\
D(2,0), D(1,1), D(0,2), D(1,0), D(0,1), D(0,0) .
\end{array}\right.
$$

Applying the differential operators to the original polynomial system $\left\{f_{1}, f_{2}, f_{3}\right\}$, we get nine new nontrivial polynomials

$$
\left\{x_{1}, x_{2}, x_{2}^{2}, 3 x_{1}^{2}+x_{2}^{2}, x_{1}+3 x_{2}, 2 x_{1} x_{2}+3 x_{2}^{2}, 2 x_{1}+2 x_{2}, 2 x_{1} x_{2}+x_{2}^{2}, x_{1}^{2}+2 x_{1} x_{2}\right\} .
$$

Add these new polynomials to $F$, the new system takes $(0,0)$ as a simple solution.

It is not a coincidence that the differential operators can be obtained from the null vectors of the coefficient matrix $M_{4}^{(0)}$.

Theorem 6 Let $Q=\left(I, P^{\rho}\right)$ be an isolated primary component of an ideal $I=\left(f_{1}, \ldots, f_{t}\right)$ at a multiple solution $\hat{\mathbf{x}}=(0, \ldots, 0)$ and $\mu$ be the multiplicity of $\hat{\mathbf{x}}$. Suppose the system $F_{\rho}=\mathrm{T}_{\rho}(F) \cup P^{\rho}$ is involutive at prolongation order $m$. The null space of the matrix $M_{\rho}^{(m)}$ is generated by vectors $\mathbf{v}_{\mathbf{1}}, \mathbf{v}_{\mathbf{2}}, \ldots, \mathbf{v}_{\mu}$. Let

$$
\mathbf{L}=[\mathbf{D}(\rho-\mathbf{1}, \mathbf{0}, \ldots, \mathbf{0}), \mathbf{D}(\rho-\mathbf{2}, \mathbf{1}, \mathbf{0}, \ldots, \mathbf{0}), \ldots, \mathbf{D}(\mathbf{0}, \ldots, \mathbf{0})]
$$

denote the vector consists of all differential operator of order less than $\rho$. Then the differential operators can be computed as

$$
L_{j}=\mathbf{L} \cdot \mathbf{v}_{\mathbf{j}}, \quad \text { for } \mathbf{1} \leq \mathbf{j} \leq \mu .
$$

Proof: Since the system $F_{\rho}$ is involutive at prolongation order $m$, for any polynomial $f \in I$, the coefficient vector $\mathbf{f}$ of the truncated polynomial $\mathrm{T}_{\rho}(f)$ can be expressed as $\mathbf{f}=\mathbf{c} \cdot \mathbf{M}_{\rho}^{(\mathbf{m})}$, where $\mathbf{c}$ is a complex vector. Then we have

$$
\left.L_{j}(f)\right|_{\mathbf{x}=\hat{\mathbf{x}}}=\left.L_{j}\left(\mathrm{~T}_{k}(f)\right)\right|_{\mathbf{x}=\hat{\mathbf{x}}}=\mathbf{c} \cdot \mathbf{M}_{\rho}^{(\mathbf{m})} \cdot \mathbf{v}_{\mathbf{j}}=\mathbf{0}, \quad \text { for } \mathbf{1} \leq \mathbf{j} \leq \mu .
$$

Algorithm 4 DifferentialOperatorsII

Input: An isolated multiple solution $\hat{x}=\left(\hat{x}_{1}, \ldots, \hat{x}_{s}\right)$ of an ideal $I=\left(f_{1}, \ldots, f_{t}\right)$ and a tolerance $\tau$.

Output: The multiplicity $\mu$, the index $\rho$ of the primary component $Q=\left(I, P^{\rho}\right)$ and differential operators $L=\left\{L_{0}, \ldots, L_{\mu-1}\right\}$. 
- Form the coefficient matrix $M_{k}^{(0)}$ by computing the truncated multivariate Taylor series expansions of $f_{1}, \ldots, f_{t}$ at $\hat{x}$ to order $k$. The prolonged matrix $M_{k}^{(j)}$ is computed by shifting $M_{k}^{(0)}$ accordingly.

- Let $d_{k}^{(j)}=\operatorname{dim} M_{k}^{(j)}$. The prolongation is stopped until $d_{k}^{(m)}=d_{k}^{(m+1)}=d_{k}$.

- If $d_{k}=d_{k-1}$, then set $\rho=k-1$ and $\mu=d_{\rho}$.

- Compute the null vectors of $M_{\rho}^{(m)}$ and denoted by $\mathbf{v}_{\mathbf{1}}, \ldots, \mathbf{v}_{\mu}$. The differential operators are computed as $L_{j}=\mathbf{L} \cdot \mathbf{v}_{\mathbf{j}}$, for $j$ from 1 to $\mu$.

Remark 3 If the zero is not at the origin, we can also compute the matrix $M_{k}^{(0)}$ by changing of variables $y_{i}=x_{i}-\hat{x}_{i}$ for $1 \leq i \leq s$ and compute the coefficients of polynomials $f_{1}\left(y_{1}+\right.$ $\left.\hat{x}_{1}, \ldots, y_{s}+\hat{x}_{s}\right), \ldots, f_{t}\left(y_{1}+\hat{x}_{1}, \ldots, y_{s}+\hat{x}_{s}\right)$ with respect to the indeterminates $y_{1}, \ldots, y_{s}$. However, we only need the coefficients of $y_{1}^{\alpha_{1}} \cdots y_{s}^{\alpha_{s}}$ with total degree less than $k$. Hence it is more efficient and numerically stable to compute the Taylor expansions at $y_{i}$ to order $k$.

There is an impressive paper written by Dayton and Zeng [9]. They compute the differential operators from the dual space. We obtain the differential operators from studying the primal space directly. These two methods could be regarded as dual to each other.

\subsection{Algorithm for Refining Approximate Singular Solution}

Suppose we are only given an approximate root

$$
\hat{\mathbf{x}}=\hat{\mathbf{x}}_{\text {exact }}+\hat{\mathbf{x}}_{\text {error }}
$$

where $\hat{\mathbf{x}}_{\text {error }}$ denotes the error in the solution and $\hat{\mathbf{x}}_{\text {exact }}$ denotes the exact solution of $F$ with multiplicity $\mu$ and $Q=\left(I, P^{\rho}\right)$ is the primary ideal defined by $\hat{\mathbf{x}}_{\text {exact }}=\left(\hat{x}_{1 \text {,exact }}, \ldots, \hat{x}_{s, \text { exact }}\right)$.

The output of the Algorithm 4 is the set of differential operators of a perturbed system of $F$ at $\hat{\mathbf{x}}$. It is not clear whether we can use these differential operators to refine the approximate solution $\hat{\mathbf{x}}$. In the following, we propose a direct method based on the prolonged matrix of an involutive system.

According to Remark 3, the output of applying the Algorithm 4 to $F$ at $\hat{\mathbf{x}}$ can be regarded as the differential operators of the perturbed system $G=\left\{g_{1}, \ldots, g_{t}\right\}$ at its approximate solution $\hat{\mathbf{y}}=\mathbf{0}$ equivalently, where

$$
g_{i}=f_{i}\left(y_{1}+\hat{x}_{1, \text { exact }}+\hat{x}_{1, \text { error }}, \ldots, y_{s}+\hat{x}_{s, \text { exact }}+\hat{x}_{s, \text { error }}\right)
$$

obtained by changing of variables $y_{i}=x_{i}-\hat{x}_{i}$, for $1 \leq i \leq s$.

We notice that the perturbed system $G$ has an exact solution

$$
\hat{\mathbf{y}}=-\hat{\mathbf{x}}_{\text {error }}=\left(-\hat{x}_{1, \text { error }}, \ldots,-\hat{x}_{s, \text { error }}\right)
$$

with the same multiplicity $\mu$ and index $\rho$. Suppose the Algorithm 4 computes multiplicity $\mu$ and index $\rho$ correctly, and the system $G_{\rho+1}$ is involutive at prolongation order $m$ for a given tolerance. Then we can use the null vectors of the matrix $M_{\rho+1}^{(m)}$ to form the multiplication matrices and compute a solution $\hat{\mathbf{y}}$ which is usually a good approximation for $-\hat{\mathbf{x}}_{\text {error }}$. 
Since the matrix $M_{\rho+1}^{(m)}$ corresponds to the coefficient matrix of $G_{\rho+1}$ by truncating the monomials $\mathbf{y}^{\alpha}$ for $\alpha \geq \rho+1$. We compute the singular solution of $G$ by forming the multiplication matrices from the truncated matrices. All computations are done modular the ideal $P^{\rho+1}=\left(y_{1}, \ldots, y_{s}\right)^{\rho+1}$. If $\hat{\mathbf{y}}$ is close to the zero, then the terms $\hat{\mathbf{y}}^{m}$ for $m>\rho$ will be much closer to zero. We are performing a generalized Newton iteration modular $P^{\rho+1}$. The profound theory still need to be investigated in future. We refer to [17] for interesting discussion on quadratic Newton iteration for systems with multiplicity.

Algorithm 5 MultipleRootRefiner

Input: An isolated approximate singular solution $\hat{\mathbf{x}}$ of an ideal $I=\left(f_{1}, \ldots, f_{t}\right)$ and a tolerance $\tau$.

Output: Refined solution $\hat{\mathbf{x}}$, the multiplicity $\mu$ and index $\rho$ of the primary component $Q=$ $\left(I, P^{\rho}\right)$ and differential operators $L=\left\{L_{0}, \ldots, L_{\mu-1}\right\}$ for the refined solution.

- For given approximate root $\hat{\mathbf{x}}$ and tolerance $\tau$, applying the Algorithm 4 to estimate the multiplicity $\mu$ and index $\rho$.

- Suppose the truncated system $G_{\rho+1}$ is involutive at prolongation order $m$, then form the multiplication matrices $M_{x_{1}}, \ldots, M_{x_{s}}$ from the null vectors of the matrix $M_{\rho+1}^{(m)} . A n$ approximate solution $\hat{\mathbf{y}}$ is obtained by averaging the trace of each multiplication matrix.

- Set $\hat{\mathbf{x}}=\hat{\mathbf{x}}+\hat{\mathbf{y}}$ and run the first two steps for the refined solution. The tolerance may be decreased according to the solution $\hat{\mathbf{y}}$.

- If $\hat{\mathbf{y}}$ converges to the origin, then we get the refined solution $\hat{\mathbf{x}}$ with high accuracy. Moreover, we apply the Algorithm 4 to compute the differential operators with respect to the refined solution. Otherwise, we may decrease the tolerance and run the above steps again.

Example 2 (continued) Suppose we are given an approximate solution $\hat{\mathbf{x}}=(0.001,-0.002)$. By changing of variables, we have a perturbed system

$$
G=\left\{f_{1}\left(y_{1}+0.001, y_{2}-0.002\right), f_{2}\left(y_{1}+0.001, y_{2}-0.002\right)\right\} .
$$

Applying the Algorithm 4 to estimate multiplicity and index for a given tolerance $\tau=10^{-2}$.

- The singular values of $M_{4}^{(0)}$ are: $\{2 ., 1 ., 1 ., 0 ., 0 ., 0 ., 0 ., 0 ., 0 ., 0$.$\} and d_{4}^{(0)}=7$.

- The singular values of $M_{4}^{(1)}$ are: $\left\{2 ., 1 ., 1\right.$, $0.004899,2.2624 \times 10^{-5}, 1.1772 \times$ $\left.10^{-5}, 8.3061 \times 10^{-6}, 5.5219 \times 10^{-22}, 8.9502 \times 10^{-24}, 0.0\right\}$ and $d_{4}^{(1)}=7$.

- The singular values of $M_{5}^{(1)}$ are: $\{2.5714,2 ., 1.6181,1.2879,1 ., 0.99999,0.8541,0.6180$, $\left.7.7620 \times 10^{-17}, 0 ., 0 ., 0 ., 0 ., 0 ., 0.\right\}$ and $d_{5}^{(1)}=7$.

- The singular values of $M_{5}^{(2)}$ are: $\{2.5714,2 ., 1.6181,1.2879,1 ., 0.99999,0.8541,0.6181$, $2.7864 \times 10^{-5}, 6.8067 \times 10^{-8}, 3.0403 \times 10^{-8}, 1.7814 \times 10^{-8}, 1.4122 \times 10^{-21}, 2.0763 \times$ $\left.10^{-22}, 1.2391 \times 10^{-25}\right\}$ and $d_{5}^{(2)}=7$. 
So that for the given tolerance $\tau=10^{-2}$, the multiplicity is $\mu=7$ and the index is $\rho=4$. The multiplication matrices are formed from the null space of $M_{5}^{(2)}$. The solution computed by averaging the eigenvalues of the multiplication matrices is:

$$
\hat{\mathbf{y}}=(-0.00099996504818215,0.0019999634232261) .
$$

Adding $\hat{\mathbf{y}}$ to $\hat{\mathbf{x}}$, we obtain the refined solution:

$$
\hat{\mathbf{x}}=(0.00000003495181785,-0.0000000365767739) \text {. }
$$

We apply the Algorithm 5 again to the new singular solution $\hat{\mathbf{x}}$ for $\tau=10^{-6}$, and get the refined solution:

$$
\hat{\mathbf{x}}=\left(1.2735 \times 10^{-17},-5.178 \times 10^{-18}\right) .
$$

\section{Experiments}

The following experiments are done for Digits $:=14$ in Maple 11 under Windows. The examples are cited from the homepage of Verschelde Jan (the references can be found there). The system D2 [8] is positive dimensional, but we can compute its isolated zero dimensional primary component at the origin. Let $Z_{t}=(-.7071, .4082, .5773, .25,-.1443,-.0482)$. We use the symbol - - to denote that the algorithm fails. The second column lists the singular solutions for the polynomial systems in the first column. We use $\rho$ and $\mu$ to represent the index and the multiplicity respectively. The fifth column consists of the results computed by the Algorithm SNEPSolver. We write down the correct digits of the perturbed zeros and the refined solutions. The sixth column records the results computed by the Algorithm MultipleRootRefiner.

\section{Conclusion}

The multiplicity structure of a singular solution has been studied extensively in $[2,21,22$, $24,7,9,8,26,25,13]$. Various methods have also been proposed for computing the singular solutions to high accuracy $[5,32,31,18,19,17]$. In this paper, we describe algorithms based on the geometric involutive form to completely describe the multiplicity structure of an isolated singular solution.

If the polynomial system and the singular solutions are known exactly, the tolerance is set to be zero. We can compute the multiplicity, index and differential operators by exact linear algebra computation. If we are given an approximate isolated singular solution of an exact polynomial system, then we can refine the singular solution to have high accuracy and obtain accurate multiplicity structure with respect to the refined solution. However, if the polynomials are only known with limited accuracy, the results we computed depend on a properly chosen tolerance. If there is no information about correct digits of the input data, we decide the tolerance by checking the biggest jump in the singular values of the coefficient matrix of the polynomial system $F$. It is interesting to investigate whether we can find a nearby polynomial system which has an isolated singular solution with given structure, such as multiplicity, index or differential operators.

Suppose the ideal $I=\left(f_{1}, \ldots, f_{t}\right)$ is zero dimensional, then applying our method to each root, we can compute the primary decomposition of $I$. However, $I$ need not to be zero 


\begin{tabular}{|c|c|c|c|c|c|}
\hline System & Zero & $\rho$ & $\mu$ & SNEPSolver & MultipleRootRefiner \\
\hline cmbs1 & $(0,0,0)$ & 5 & 11 & $5 \rightarrow 14$ & $3 \rightarrow 11 \rightarrow 15$ \\
\hline cmbs2 & $(0,0,0)$ & 4 & 8 & $5 \rightarrow 15$ & $3 \rightarrow 13 \rightarrow 15$ \\
\hline mth191 & $(0,1,0)$ & 3 & 4 & $5 \rightarrow 10 \rightarrow 15$ & $4 \rightarrow 9 \rightarrow 15$ \\
\hline LVZ & $(0,0,-1)$ & 7 & 18 & -- & $5 \rightarrow 10 \rightarrow 14$ \\
\hline KSS & $(1,1,1,1,1,1)$ & 5 & 16 & -- & $5 \rightarrow 11 \rightarrow 14$ \\
\hline Caprasse & $(2,-i \sqrt{3}, 2, i \sqrt{3})$ & 3 & 4 & $5 \rightarrow 14$ & $4 \rightarrow 12 \rightarrow 15$ \\
\hline DZ1 & $(0,0,0,0)$ & 11 & 131 & $5 \rightarrow 14$ & $5 \rightarrow 14$ \\
\hline DZ2 & $(0,0,-1)$ & 8 & 16 & -- & $4 \rightarrow 7 \rightarrow 14$ \\
\hline tangens & $Z_{t}$ & 4 & 4 & -- & $3 \rightarrow 10 \rightarrow 16$ \\
\hline D2 & $(0,0,0)$ & 5 & 5 & $5 \rightarrow 10 \rightarrow 15$ & $5 \rightarrow 10 \rightarrow 15$ \\
\hline Ojika1 & $(1,2)$ & 3 & 3 & $5 \rightarrow 7 \rightarrow 14$ & $3 \rightarrow 6 \rightarrow 18$ \\
\hline Ojika2 & $(0,1,0)$ & 2 & 2 & $5 \rightarrow 10 \rightarrow 15$ & $5 \rightarrow 10 \rightarrow 14$ \\
\hline Ojika3 & $(0,0,1)$ & 3 & 4 & $5 \rightarrow 9 \rightarrow 14$ & $4 \rightarrow 8 \rightarrow 15$ \\
\hline Ojika4 & $(0,0,1)$ & 3 & 3 & $5 \rightarrow 10 \rightarrow 14$ & $3 \rightarrow 7 \rightarrow 15$ \\
\hline
\end{tabular}

Table 1. Algorithm Performance

dimensional. We only require that the primary component $Q$ is isolated and zero dimensional. Furthermore, the algorithms we present can be generalized to compute an isolated primary component for an isolated maximal ideal represented by polynomials, not only the ones generated by the singular solutions.

\section{Acknowledgments}

We greatly thank Greg Reid for fruitful discussions.

\section{References}

[1] Auzinger, W. and Stetter, H., Polynomial Factorization, Intern. Series in Numer. Math., 86:1130, 1988 .

[2] Bates, Dan, Peterson, Chris, and J.Sommese, Andrew, A Numerical-Symbolic Algorithm for Computing the Multiplicity of a Component of an Algebraic Set, Journal of Complexity, 22:475489, 2006.

[3] Bayer, David and Stillman, Michael, A Criterion for Detecting m-regularity, Inventiones Mathematicae, 87(1):1-11, 1987.

[4] Bonasia, J., Lemaire, F., Reid, G.J., Scott, R., and zhi, L., Determination of Approximate Symmetries of Differential Equations, In CRM Proceedings and Lecture Notes, 233-249, 2004.

[5] Corless, R.M., Gianni, P.M., and Trager, B.M., A Recorded Schur Factorization Method for Zero-dimensional Polynomial Systems with Multiple Roots, Proc. ISSAC1997, 133-140, 1997.

[6] Cox, David, Little, John, and Shea, Donal O', em Ideals, Varieties, and Algorithms, SpringerVerlag, New York, 1992. 
[7] Damiano, Alberto, Sabadini, Irene, and C.Struppa, Daniele, Computational Methods for the Construction of a Class of Noetherian Operators, Experiment. Math., 16:41-55, 2007.

[8] Dayton, B.H., Numerical Local Rings and Local Solutions of Nonlinear Systems, Proc. SNC2007,79-86, 2007.

[9] Dayton, B.H. and Zeng, Z., Computing the Multiplicity Structure in Solving Polynomial Systems, Proc. ISSAC2005, 116-123, 2005.

[10] Faugère, J.C., A New Efficient Algorithm for Computing Gröbner Bases without Reduction to Zero, Proc. ISSAC2002, 75-83, 2002.

[11] Gerdt, V.P. and Blinkov, Y.A.,Involutive Bases of Polynomial Ideals, Mathematics and Computers in Simulation, 45:519-541, 1998.

[12] Gianni, Patrizia, Trager, Barry, and Zacharias, Gail, Gröbner Bases and Primary Decomposition of Polynomial Ideals, J. Symbolic computation, 6:149-167, 1988.

[13] Kobayashi, H., Suzuki, H., and Sakai, Y., Numerical Calculation of the Multiplicity of a Solution to Algebraic Equations, Math. Comp., 67(221):257-270, 1998.

[14] Kuranishi,M., On E.Cartans's Prolongation Theorem of Exterior Differential Systems, Amer. J. Math., 79:1-47, 1957.

[15] Lakshman, Y.N., A single Exponential Bound on the Complexity of Computing Gröbner Bases of Zero Dimensional Ideals, Effective Methods in Algebraic Geometry, Progress in Mathematics, 227-234, 1994.

[16] Lazard, D., Gröbner Bases, Gaussian Elimination and Resolution of Systems of Algebraic Equations, Proc. European Conf. Comput. Algebra. Lect. Notes in Comp. Sci., 146-157, 1983.

[17] Lecerf, G., Quadratic Newton Iteration for Systems with Multiplicity, Foundations of Computational Mathematics, 2(3):247-293, 2002.

[18] Leykin, Anton, Verschelde, Jan, and Zhao, Ailing, Newton's Method with Deflation for Isolated Singularities of Polynomial Systems, Theoretical Computer Science, 359:111-122, 2006.

[19] Leykin, Anton, Verschelde, Jan, and Zhao, Ailing, Higher-order Deflation for Polynomial Systems, Manuscript, 19 pages, 2007.

[20] Malgrange, B., Cartan Involutiveness = Mumford Regularity, contemp. Math., 331:193-205, 2003.

[21] Marinari, Maria Grazia, Mora, Teo, and Möller, Hans Michael, Gröbner Duality and Multiplicities in Polynomial Solving, Proc. ISSAC1995, 167-179, 1995.

[22] Marinari, Maria Grazia, Mora, Teo, and Möller, Hans Michael, On Multiplicities in Polynomial System solving, Trans. Amer. Math. Soc., 348:3283-3321, 1996.

[23] Möller, H.M. and Sauer, T., H-bases for Polynomial Interpolation and System solving, Advances Comput. Math., 12:23-35, 2000.

[24] Möller, H.M. and Stetter, H., Multivariate Polynomial Equations with Multiple Zeros Solved by Matrix Eigenspaces, Numer. Math., 70:311-329, 1995.

[25] Möller, Hans Michael and Tenberg, Ralf, Multivariate Polynomial System Solving Using Intersections of Eigenspaces, J. Symbolic Computation, 30:1-19, 2001.

[26] Mourrain, B., Isolated Points, Duality and Residues, J. of Pure and Applied Algebra, 469-493, 1996.

[27] Mourrain, B., A New Criterion for Normal Form Algorithms, AAECC, 1719:430-443, 1999.

[28] Mourrain, B. and Trébuchet, P., Solving Projective Complete Intersection Faster, Proc. ISSAC2000, 430-443, 2000.

[29] Mourrain, B. and Trébuchet, P., Algebraic Methods for Numerical Solving, Proc. the 3rd International Workshop on Symbolic and Numeric Algorithms for Scientific Computing, 42-47, 2002. 
[30] Mourrain, B. and Trébuchet, P., Generalized Normal Forms and Polynomial System Solving, Proc. ISSAC2005, 253-260, 2005.

[31] Ojika, T., Modified Deflation Algorithm for the Solution of Singular Problems, J. Math. Anal. Appl., 123:199-221, 1987.

[32] Ojika, T., Watanabe, S., and Mitsui, T., Deflation Algorithm for the Multiple Roots of a System of Nonlinear Equations, J. math. anal. Appl., 96:463-479, 1983.

[33] Pommaret, J.F., Systems of Partial Differential Equations and Lie Pseudogroups, Gordon and Breach Science Publishers, 1978.

[34] Reid, Greg, Scott, Robin, Wu, Wenyuan, and Zhi, Lihong, Algebraic and Geometric Properties of Nearby Projectively Involutive Polynomial Systems, Manuscript, 2005.

[35] Reid, G.J., Tang, J., and Zhi, L., A Complete Symbolic-numeric Linear Method for Camera Pose Determination, Proc. ISSAC2003, 215-223, 2003.

[36] Reid, Greg and Zhi, Lihong, Solving Polynomial Systems via Symbolic-numeric Elimination Method, Manuscript, 2007.

[37] Seiler, W.M., Involution - The Formal Theory of Differential Equations in Computer Algebra and Numerical Analysis, Habilitation thesis, Univ. of Mannheim, Germany, 2002.

[38] Stetter, H.J., Numerical Polynomial Algebra, SIAM, Philadelphia, 2004.

[39] Trébuchet, P., Vers une Résolution Stable et Rapide des Équations Algébriques, Université Pierre et Marie Curie, France, 2002.

[40] van der Waerden B.L., Algebra, Frederick Ungar Pub. Co., 1970.

[41] Wittkopf, A.D. and Reid, G.J., Fast Differential Elimination in C: The CDiffelim Environment, Comp. Phys. Comm., 139(2):192-217, 2001.

[42] Zhi, Lihong and Reid, Greg, Solving Nonlinear Polynomial System via Symbolic-numeric Elimination Method, Proc. International Conference on Polynomial System Solving, 50-53, 2004. 